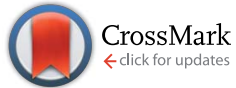

Cite this: RSC Adv., 2016, 6, 43724

Received 2nd March 2016 Accepted 16th April 2016

DOI: 10.1039/c6ra05553b

www.rsc.org/advances

\section{A novel sensitive and selective electrochemical sensor based on molecularly imprinted polymer on a nanoporous gold leaf modified electrode for warfarin sodium determination $\uparrow$}

\begin{abstract}
Yingchun Li, ${ }^{* a b}$ Lu Zhang, ${ }^{a}$ Jiang Liu, ${ }^{a}$ Shu-Feng Zhou, ${ }^{d}$ Khalid Abdullah Al-Ghanim, ${ }^{e}$ Shahid Mahboob, ${ }^{e}$ Bang-Ce $\mathrm{Ye}^{\mathrm{b}}$ and Xueji Zhang ${ }^{* c}$

Warfarin sodium (WFS) is a widely used oral anticoagulant drug but has a narrow therapeutic window. Since traditional detection methods applied for therapeutic drug monitoring suffer some shortcomings, including difficulty in obtaining timely reports, high costs and tedious operation, it is necessary to develop a detection system for the rapid monitoring of WFS in biological samples. Here we report a novel electrochemical sensor, which was facilely fabricated by coupling nanoporous gold leaf (NPGL) with molecularly imprinted polymer (MIP) to afford ultrasensitive and selective determination of WFS. The morphological characterization via scanning electron microscopy proved the successful modification of the sensor by NPGL followed with MIP layer modification. The influencing parameters including the type of monomer, $\mathrm{pH}$ and molar ratio of template to monomer were optimized during electro-polymerization. Using $\mathrm{Fe}(\mathrm{CN})_{6}{ }^{3-14-}$ as a probe acting as an electrical indicator, a linear relationship of the current response versus the concentration of WFS was obtained in the range from $1.0 \times 10^{-10}$ to $8.0 \times 10^{-8} \mathrm{M}$ under the optimal experimental conditions, with a detection limit of $4.1 \times 10^{-11} \mathrm{M}(\mathrm{S} / \mathrm{N}=3)$. In addition, the asprepared sensor exhibited specific detection of WFS over its structural analogues and interferents, and the established electrochemical approach was validated using the standard method - high performance liquid chromatography. Eventually, rapid and accurate determination of WFS in human blood was carried out after easy sample pretreatment.
\end{abstract}

\section{Introduction}

Warfarin sodium (WFS), a coumarin derivative, is an oral anticoagulant widely used in multifarious cerebrovascular and cardiovascular disorders, for instance, venous pulmonary embolism, atrial fibrillation, coronary heart diseases, etc. Despite its effectiveness, treatment with warfarin has several shortcomings, one of which is a narrow therapeutic window. Dosage out of the window may cause either treatment failure or

${ }^{a}$ School of Pharmacy, Shihezi University, Shihezi 832000, China. E-mail: yingchunli@ shzu.edu.cn; Fax: +86-993-2057005; Tel: +86-993-2057005

${ }^{b}$ Key Laboratory for Green Processing of Chemical Engineering of Xinjiang Bingtuan, School of Chemistry and Chemical Engineering, Shihezi University, Shihezi 832003, China

${ }^{c}$ Research Center for Bioengineering and Sensing Technology, University of Science and Technology Beijing, Beijing 100083, China.E-mail: zhangxueji@ustb.edu.cn; Fax:+8610-62333910; Tel: +86-10-62333910

${ }^{d}$ Department of Pharmaceutical Science, College of Pharmacy, University of South Florida, Tampa, FL 33612, USA

${ }^{e}$ Department of Zoology, College of Science, King Saud University, P.O. Box 2455, Riyadh-11451, Saudi Arabia

$\dagger$ Electronic supplementary information (ESI) available. See DOI: 10.1039/c6ra05553b unwanted bleeding and even threat to life. Therefore, the activity of WFS has to be monitored by blood testing to ensure an adequate yet safe dose..$^{\mathbf{1 - 4}}$

The methods of determining WFS in biological samples include high performance liquid chromatography (HPLC) using fluorescence ${ }^{5}$ or ultraviolet detectors, ${ }^{6}$ liquid chromatographytandem mass spectrometry (LC-MS/MS), ${ }^{7}$ capillary zone electrophoresis (CZE), ${ }^{8}$ and so on. However, particular concerns for these large instrument-based strategies are high energy and money consumption, a long working time, tedious pretreatment, etc., which is especially unsuitable for fast therapeutic drug monitoring. An alternative to the aforementioned techniques is electrochemical sensing, which gains much attention owing to easy preparation, high sensitivity, low detection limit, etc. ${ }^{9,10}$ Specificity, however, is a common problem for sensorbased determination since normally no separation system is involved in the detection procedure. Therefore, physical, chemical or biological modification is often necessary to endow a sensor with a specific recognition ability toward target molecules. ${ }^{11-14}$ A very promising modifier candidate is molecularly imprinted polymer (MIP) since it is able to provide antibodylike specificity and long-term stability. ${ }^{15}$ Another barrier 
encountered in electrochemical sensing is that analytes must have electrochemical activity, ${ }^{16-21}$ which means that the redox reaction of the target analyte should happen at the sensor surface under certain experimental conditions. As redox reaction conditions for different analytes could be very different, tedious optimization work is often needed in order to find suitable conditions and achieve sensitive electrical signals. In our previous work, we have developed a versatile way of measuring various substances by introducing active probes as electrical signal indicators (e.g. ferri/ferro-cyanide) along with an MIP-modified electrochemical sensor..$^{22-24}$ Such a strategy is independent of the electrochemical activity of the analyte itself, and thus it is possible to test any species using an electrochemical sensor.

Apart from specificity and feasibility, another important issue in sensor development is sensitivity. As materials with nanostructures are expected to enlarge the effective surface area of a planar sensor surface, various nano-agents have been employed to modify sensors for the purpose of improving sensitivity. ${ }^{25-30}$ Among them, a very suitable material is nanoporous gold leaf (NPGL). The unique features of NPGL include high conductivity, interconnected three-dimensional (3D) architecture, uniformly distributed nanopores and nanoligaments, good biocompatibility, a relatively low cost, and possibility for mass production. ${ }^{31-33}$ Compared with gold nanoparticles, NPGL is a free-standing mesoporous thin film with a rigid 3D framework structure which avoids particle stacking, thus assuring controllability and stability of the NPGL modified sensor. In the present work, we fabricated an electrochemical sensor by combining NPGL and MIP and furthermore the composite-film decorated sensor was applied for the detection of WFS. Several parameters influencing the sensing performance have been carefully optimized and the resulting sensor has been successfully adopted to analyze WFS in human blood.

\section{Experimental}

\subsection{Materials and apparatus}

Warfarin sodium (WFS), aspirin (ASP), hydrochlorothiazide (HCT), and vitamin $\mathrm{K}_{4}\left(\mathrm{VK}_{4}\right)$ were obtained from J\&K Scientific Ltd. (Beijing, China). Phenylenediamine, resorcinol and $o$ aminophenol were purchased from Shanghai Aladdin Co. (Shanghai, China). Dopamine was obtained from Adamas Reagent Co. Ltd. Au/Ag alloy leaves (50:50 wt\%; $100 \mathrm{~nm}$ in thickness) were purchased from Suzhou ColdStones Tech. Co. Ltd. (Suzhou, China). Human blood samples were obtained from the first affiliated hospital of the medical college of Shihezi University (Shihezi, China). Reagents and materials, such as starch, sucrose, dextrin glucose, $\mathrm{Fe}(\mathrm{CN})_{6}{ }^{3-/ 4-}, \mathrm{NaCl}, \mathrm{CaCl}_{2}$, $\mathrm{NH}_{4} \mathrm{Cl}, \mathrm{H}_{2} \mathrm{SO}_{4}, \mathrm{KNO}_{3}, \mathrm{HNO}_{3}$, methanol and phosphate buffer solution (PBS, $\mathrm{KH}_{2} \mathrm{PO}_{4}$ and $\mathrm{K}_{2} \mathrm{HPO}_{4}$ ) were of analytical grade.

Electrochemical measurements were performed on a $\mathrm{CHI}$ $760 \mathrm{E}$ Electrochemical Workstation (CHI Instruments Co., Shanghai, China) connected to a PC at room temperature. A conventional three-electrode system was employed, consisting of a bare or a modified planar gold electrode (GE, $4 \mathrm{~mm}$ in diameter) as the working electrode, a saturated calomel electrode (SCE) as the reference electrode and a platinum wire (0.5 $\mathrm{mm}$ in diameter, $34 \mathrm{~mm}$ in length) as the counter electrode. All potentials given in this paper were referenced to the SCE. The surface morphology of the NPGL was characterized using a Zeiss Supra55VP scanning electron microscope (SEM) operating at $20 \mathrm{kV}$. HPLC was performed with a Shimadzu (Japan) system comprising of LC-10A pumps and an SPD-10A UVdetector. The LC conditions were as follows: chromatographic separation was performed on a WondaSil $\mathrm{C}_{18}$ column $(150 \mathrm{~mm}$ $\times 4.6 \mathrm{~mm}$ i.d., $5 \mu \mathrm{m}$ ) which was purchased from Dalian Elite Analytical Instruments Co. (Dalian, China). The mobile phase for WFS was acetonitrile-methanol-water $(70: 30: 1, \mathrm{v} / \mathrm{v} / \mathrm{v})$ with

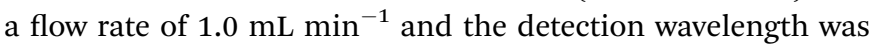
$308 \mathrm{~nm}$. All experiments involving the use of human subjects were performed in compliance with the relevant laws and institutional guidelines, and the institutional committees have approved the experiments. The informed consent was obtained for any experimentation with human subjects.

\subsection{Preparation of sensor}

The $\mathrm{Ag} / \mathrm{Au}$ alloy leaves were pruned into a $7 \times 7 \mathrm{~mm}$ sheet and floated onto the surface of $65 \mathrm{wt} \%$ nitric acid. Erosion was stopped after $60 \mathrm{~min}$, and then the sample was washed with distilled water thoroughly and the NPGL was obtained. GE was first polished repeatedly to a mirror finish with 0.3 and $0.05 \mathrm{~mm}$ $\mathrm{Al}_{2} \mathrm{O}_{3}$ and thoroughly cleaned with distilled water before use. NPGL was carefully affixed to the GE surface and the NPGLmodified GE (NPGL/GE) was dried under an infrared lamp for $15 \mathrm{~min}$. The MIP was electro-polymerized on the electrode surface using cyclic voltammetry (CV), which was performed between 0 and $1.0 \mathrm{~V}$ ( $v s$. SCE) for 15 cycles at a scan rate of $50 \mathrm{mV}$ $\mathrm{s}^{-1}$ in a solution containing functional monomers and WFS. Afterwards, the polymer film-covered NPGL/GE was immersed in $0.1 \mathrm{M} \mathrm{NaOH}$ to remove embedded WFS by scanning from $-0.5 \mathrm{~V}$ to $+0.5 \mathrm{~V}$ for several cycles until obvious and stable redox peaks could be found in the probe solution of $5 \mathrm{mM} \mathrm{Fe}(\mathrm{CN})_{6}{ }^{3-1}$ ${ }^{4-}$ and $0.1 \mathrm{M} \mathrm{KCl}$. A schematic representation for the preparation of WFS-MIP/NPGL/GE is shown in Fig. 1. For comparison, a non-imprinted polymer (NIP)-modified electrode (NIP/NPGL/

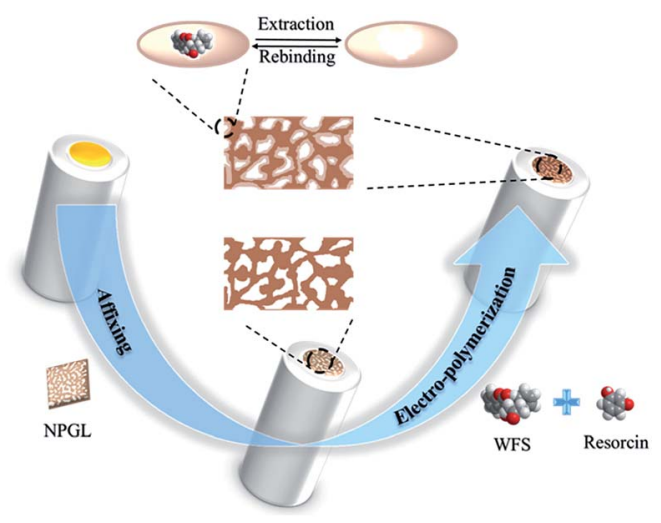

Fig. 1 Schematic representation of WFS-MIP/NPGL/GE fabrication. 

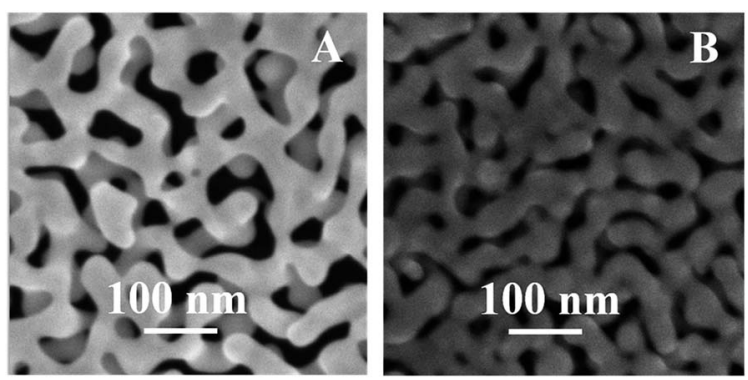

Fig. 2 SEM images of (A) an NPGL-modified electrode and (B) an MIPNPGL-modified electrode.

GE) was prepared using the same method but in the absence of the template-WFS during electro-polymerization. ${ }^{34}$

\subsection{Electrochemical measurement}

The electrochemical behavior of different electrodes was studied in the probe solution using $\mathrm{CV}$, which was operated in the scanning range of -0.2 to $+0.6 \mathrm{~V}$ at a scan rate of $100 \mathrm{mV}$ $\mathrm{s}^{-1}$. A sensor was first incubated in a sample solution containing the analyte for $10 \mathrm{~min}$, after which the electrode was washed with water and applied for voltammetric measurement. The peak current shift $(\Delta I)$ was calculated from the reduction peak currents of $\mathrm{Fe}(\mathrm{CN})_{6}{ }^{3-/ 4-}$ obtained before and after binding with WFS. The shift was used to explore the influence of different modifications on the sensor performance and to establish a calibration curve for sample determination. After each analysis, the sensor was recovered by immersion in $0.1 \mathrm{M} \mathrm{NaOH}$ with $\mathrm{CV}$ scanning between -0.5 to $+0.5 \mathrm{~V}$ for several cycles to remove WFS at the electrode surface. The electrode was reusable after this cleaning procedure. Electrochemical impedance spectroscopy (EIS) experiments were carried out in a solution containing $5 \mathrm{mM} \mathrm{Fe}(\mathrm{CN})_{6}{ }^{3-/ 4-}$ and $0.1 \mathrm{M} \mathrm{KCl}$ within a frequency range from $0.01 \mathrm{~Hz}$ to $100 \mathrm{kHz}$. All of the electrochemical experiments were performed at room temperature.

\subsection{Detection of WFS in biological samples}

A certain amount of WFS was added into human blood samples for a spiked recovery experiment. The sample was centrifuged at $4000 \mathrm{rpm}$ for $10 \mathrm{~min}$ and methanol was added into the supernatant at a volumetric ratio of $1: 1$, followed by further centrifugation at $4000 \mathrm{rpm}$ for $10 \mathrm{~min}$ to get rid of protein. The final supernatant was used for WFS detection.

\section{Results and discussion}

\subsection{Preparation and characterization of WFS-MIP/NPGL/GE electrode}

The process of preparing a hybrid sensor modified with MIP and NPGL composite film is illustrated in Fig. 1. The morphology of the electrode surface was characterized using SEM, and it exhibits a sponge-like conformation with metal ligaments and nanopore channels (Fig. 2A). The modification of NPGL with an electro-synthesized MIP layer is obvious as the width of the nanopores decreases from $\sim 30 \mathrm{~nm}$ to $\sim 20 \mathrm{~nm}$ and the image gets darker compared with bare NPGL (Fig. 2B). Ag/ $\mathrm{Au}$ alloy leaves, NPGL and molecularly imprinted polymer decorated NPGL (MIP/NPGL) after the removal of WFS were probed via energy dispersive spectroscopy (EDS) and element mapping. All of the related information is given in Fig. S1-S4 in the ESI file. $\uparrow$ Compared with the EDS spectrum of $\mathrm{Ag} / \mathrm{Au}$ alloy leaf (Fig. S1a $\dagger$ ), there are only Au signals in NPGL (Fig. S1b $\dagger$ ), indicating that $\mathrm{Ag}$ was removed after dealloying. When MIP covered the surface of NPGL, the elements carbon and oxygen can be observed from the EDS spectra (Fig. S1c $\dagger$ ), and their relative contents correspond to the calculated values of the functional monomer (resorcin, $\mathrm{C}_{6} \mathrm{H}_{6} \mathrm{O}_{2}$ ), which confirms the formation of an MIP network on NPGL. From the element mapping images (Fig. S2 to S4†), one can see that the elements $\mathrm{Au}, \mathrm{C}$ and $\mathrm{O}$ are uniformly distributed on the electrode surface, implying that a homogeneous MIP film was obtained on NPGL by the in situ electropolymerization of the functional monomers. The electrochemical behavior of the stepwise production
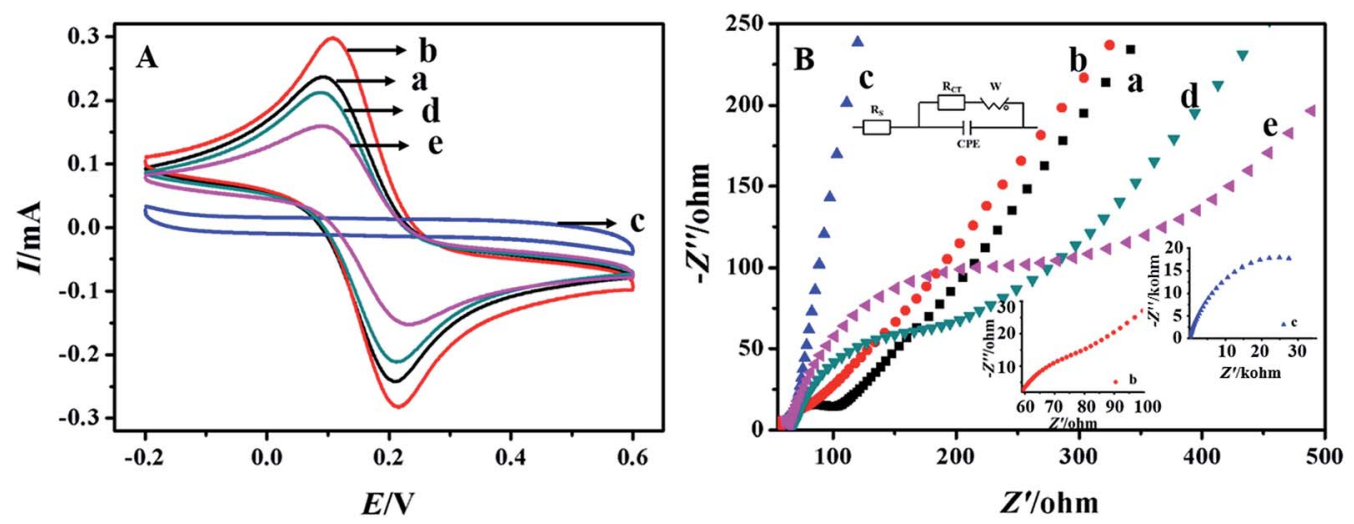

Fig. 3 Cyclic voltammograms (A) and Nyquist diagrams of EIS (B) for bare GE (a), NPGL/GE (b), MIP/NPGL/GE before extraction of WFS (c), MIP/ NPGL/GE after extraction of WFS (d) and MIP/NPGL/GE after rebinding of WFS (e). The insets of (B) are the EIS plots for (b) and (c) in their proper coordinate ranges and the equivalent circuit diagram. The supporting electrolyte of $\mathrm{CV}(\mathrm{A})$ contained $0.1 \mathrm{M} \mathrm{Fe}(\mathrm{CN})_{6}{ }^{3-14-}$ and $0.1 \mathrm{M} \mathrm{KNO}{ }_{3}$, and that of EIS (B) contained $5 \mathrm{mM} \mathrm{Fe}(\mathrm{CN})_{6}^{3-/ 4-}$ and $0.1 \mathrm{M} \mathrm{KCl}$. The scan rate of $\mathrm{CV}(\mathrm{A})$ was $100 \mathrm{mV} \mathrm{s}^{-1}$ and the frequency range of EIS (B) was from $0.01 \mathrm{~Hz}$ to $100 \mathrm{kHz}$. 

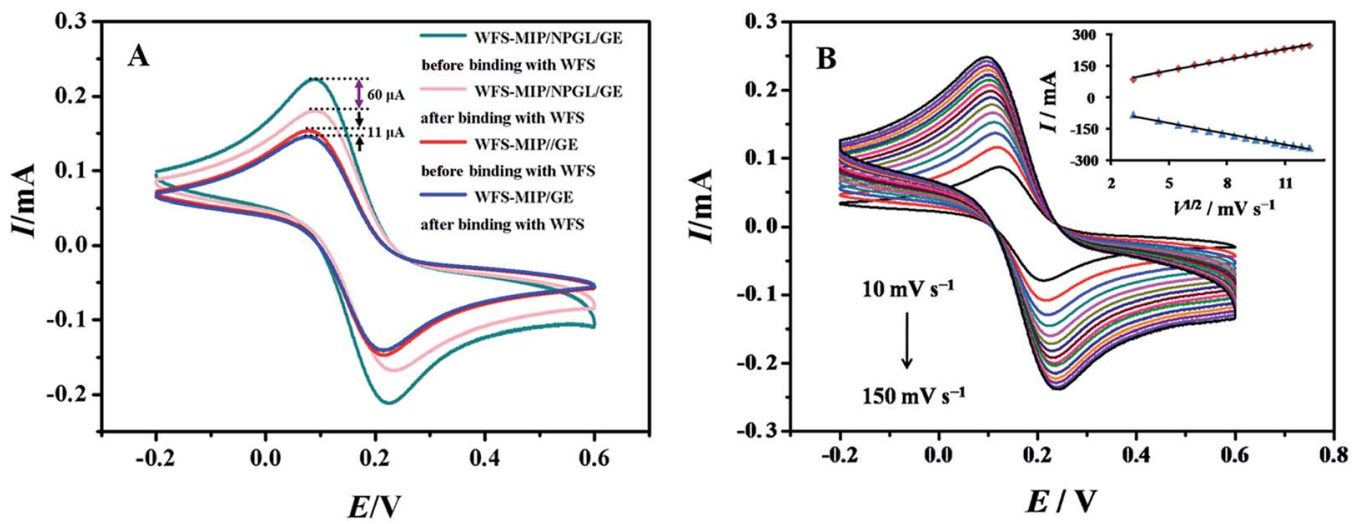

Fig. 4 Cyclic voltammograms of (A) MIP/NPGL/GE and MIP/GE at a scan rate of $100 \mathrm{mV} \mathrm{s}^{-1}$ before and after binding with WFS and of (B) $\mathrm{MIP} / \mathrm{NPGL} / \mathrm{GE}$ at the scan rate from 10 to $150 \mathrm{mV} \mathrm{s}^{-1}$. The electrolyte contained $5 \mathrm{mM} \mathrm{Fe}(\mathrm{CN})_{6}{ }^{3-/ 4-}$ and $0.1 \mathrm{M} \mathrm{KCl}$; the inset of $(\mathrm{B})$ is the anodic and the cathodic peak currents versus the square-root of the scan rate plot.

process was investigated via $\mathrm{CV}$ in a probe solution. As shown in Fig. 3A, compared with bare GE (curve a), the peak currents of NPGL/GE (curve b) increase obviously due to its enlarged surface area, which can enhance the detection sensitivity. After MIP modification, the redox peaks of $\mathrm{Fe}(\mathrm{CN})_{6}{ }^{3-14-}$ disappear (curve c). This phenomenon can be explained by MIP not being conductive and after the whole electrode surface was densely covered with the polymeric film, there was virtually no channel for the active probe ions to access the electrode surface. This also proves the successful preparation of WFS-MIP film on the entire 3D surface of the NPGL electrode. After that, the removal of the template leads to an increase of the peak currents (curve d), as the cavities generated in the rigid polymer matrix open doors for the probe ions to transfer to the electrolyte/electrode interface. When the electrode was immersed in the solution of WFS, the rebinding of WFS by MIP impeded the electron transportation of $\mathrm{Fe}(\mathrm{CN})_{6}{ }^{3-/ 4-}$, resulting in current reduction (curve e).

Electrochemical impedance spectroscopy (EIS) is also a helpful way of characterizing the stepwise sensor construction

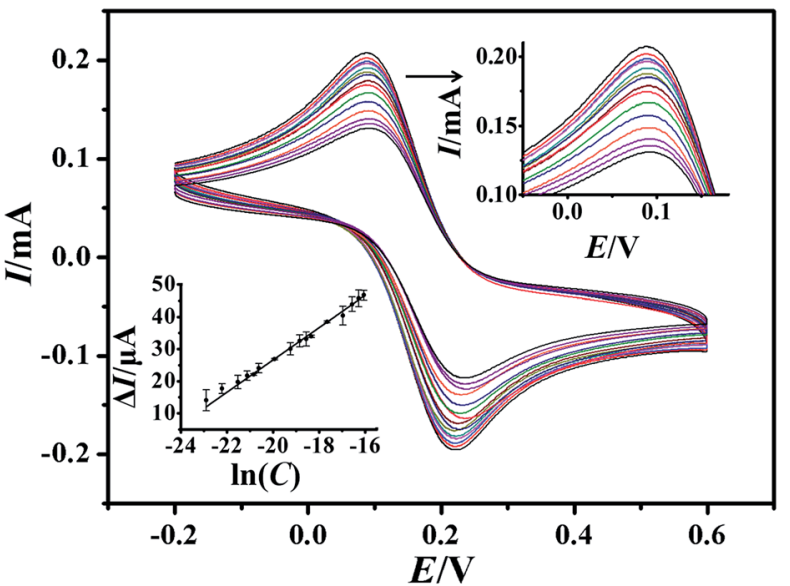

Fig. 6 Cyclic voltammograms of MIP/NPGL/GE in the probe solution after rebinding with WFS in the concentration range of $1 \times 10^{-10}$ to 8 $\times 10^{-8} \mathrm{M}$. The top-right inset shows partially amplified cyclic voltammograms and the bottom-left inset is the calibration curves correlating current shift $(\Delta /)$ with the logarithm of concentration of WFS $(\ln C)$.
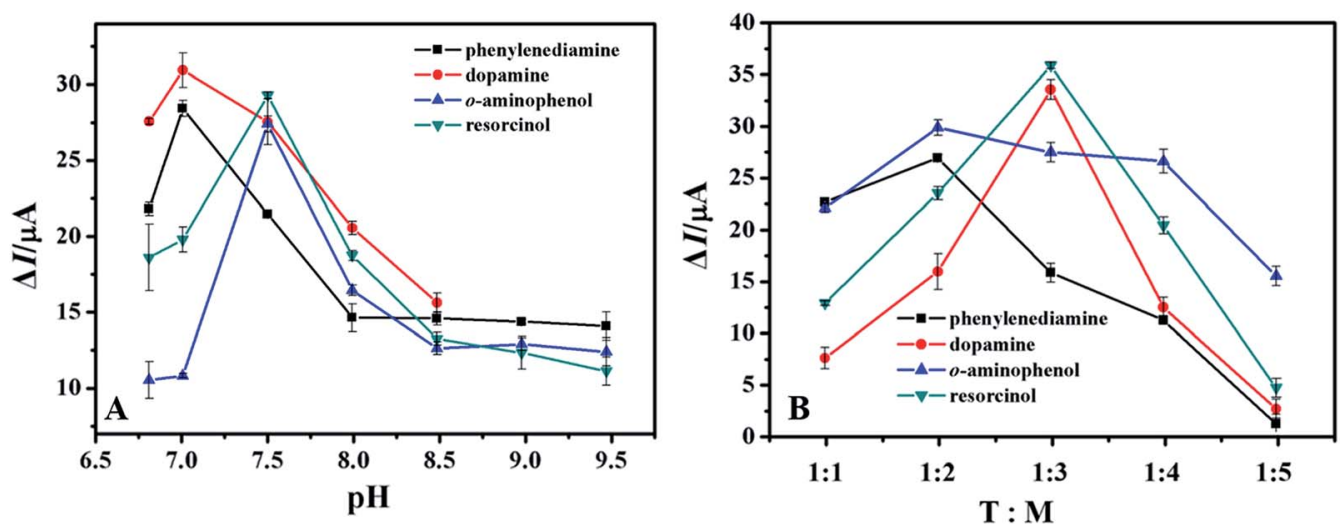

Fig. 5 Sensing responses toward 1.0 nM WFS using different MIP/GE prepared with varied monomers and pH values (A) and with varied ratios of template and monomer $(T: M)$ under the individual optimal $\mathrm{pH}$ values (B). The $T: M$ was set at $1: 3$ for $(A)$. All the experiments were repeated in triplicate. 
Table 1 Comparison of the major characteristics of some electrochemical methods for WFS detection ${ }^{h}$

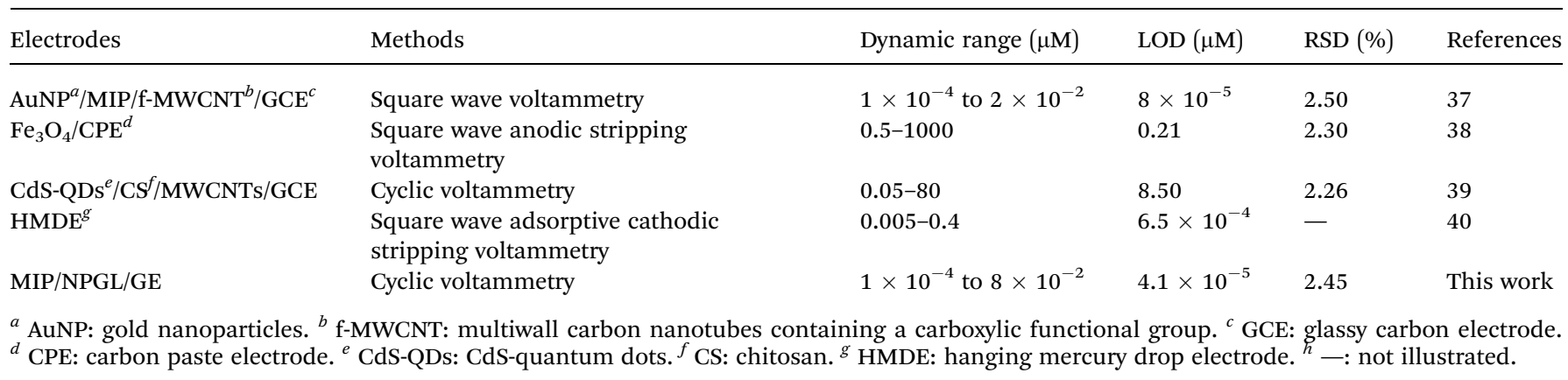

process and provides useful information on impedance change at the electrode/electrolyte interface. ${ }^{35}$ Fig. $3 \mathrm{~B}$ depicts the EIS diagrams of electrodes at each preparation step. Bare GE (curve a) yielded a small semicircle, implying that the electrode has quite good conductivity. In comparison, the semicircle at NPGL/ GE (curve b) got much smaller, suggesting that the nanoporous structure of NPGL benefits electron migration effectively. A significant increase in the diameter of the semicircle was observed in curve $\mathrm{c}$, which is ascribed to MIP modification and the polymer acting as a compact barrier for electron transfer. The conductivity of the electrode was recovered by getting rid of the template (curve d) due to the cavities created in the polymer matrix. Finally, the rebinding of WFS increased the impedance of the electrode (curve e), as a result of WFS embedding in binding sites and consequently inhibiting the redox reactions of probe ions.

In order to investigate the role of NPGL on sensor performance, MIP/GE was prepared as a comparison sensor. Fig. 4A shows the cyclic voltammograms of MIP/NPGL/GE and MIP/GE before and after binding with WFS, showing that the current shift of MIP/NPGL/GE is about 5.3 times larger than that of MIP/ GE. The enhanced sensing response of the hybrid sensor can be ascribed to the nanoporous architecture of NPGL which enhanced the active surface area and the conductivity of the electrode.

Fig. 4B depicts the change in the cyclic voltammograms of MIP/NPGL/GE with varied scan rates. A linear relationship was observed between the redox peak currents and the square root of the scan rate, revealing a diffusion-controlled mechanism at the sensor surface. ${ }^{36}$

\subsection{Optimization of MIP/NPGL/GE preparation}

3.2.1 Influence of $\mathrm{pH}$ value on electro-polymerization of MIP. Four monomers (phenylenediamine, dopamine, $o$-aminophenol and resorcinol) were tested as functional monomer candidates for achieving the optimal imprinting effect toward WFS. First, the $\mathrm{pH}$ values during polymerization were optimized for each monomer with a molar ratio of template to monomer ( $\mathrm{T}: \mathrm{M})$ of $1: 3$. Since the precipitation of WFS occurs when the $\mathrm{pH}$ value is less than 6.8 , the $\mathrm{pH}$ range in the optimization work started at 6.8. After polymerization and the extraction of WFS, the electrodes modified with different MIP films were employed for detection of WFS at the same concentration of $1.0 \times 10^{-9} \mathrm{M}$ and their reduction peak current shifts $(\Delta I)$ before and after the rebinding of WFS were used to evaluate the individual response level. As shown in Fig. 5A, the optimum $\mathrm{pH}$ values for phenylenediamine, dopamine, $o$-aminophenol and resorcinol are 7.5, $7.0,7.5$ and 7.0, respectively.

3.2.2 Optimization of the molar ratio of template to monomer $(\mathbf{T}: \mathbf{M})$. After choosing the most ideal $\mathrm{pH}$ value for each monomer, the molar ratio between the template molecule and monomer $(\mathrm{T}: \mathrm{M})$ was studied in the ratio range from $1: 1$ to $1: 5$. The effect of $\mathrm{T}: \mathrm{M}$ is related to the number of binding sites generated during MIP preparation. Inadequate functional monomer results in insufficient binding sites available for WFS binding, while an excessive amount of monomer during
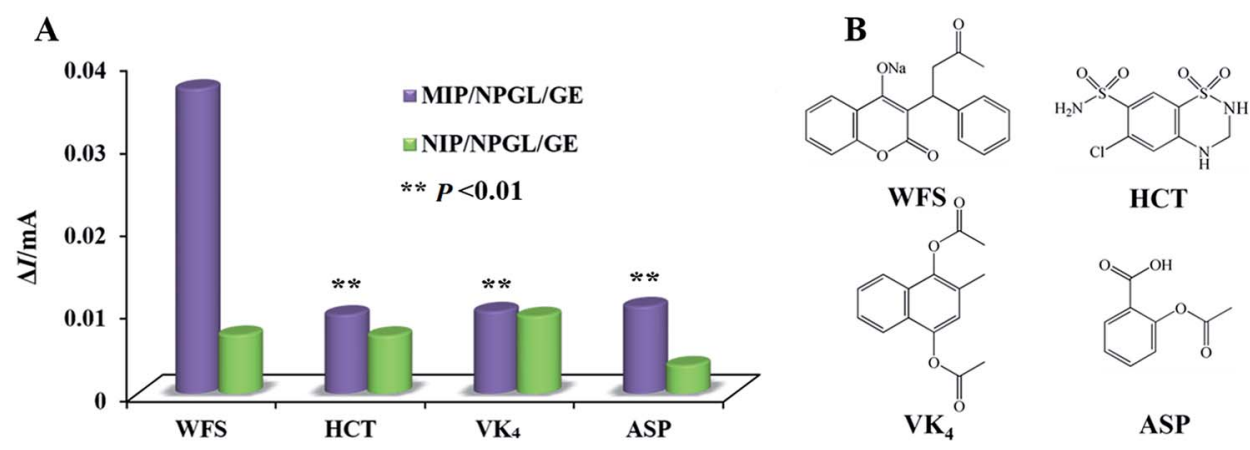

Fig. 7 Comparison of the sensor responses towards WFS and its structural analogues at the same concentration of $8 \times 10^{-9} \mathrm{M}$ using MIP/NPGL/ GE and NIP/NPGL/GE (A); chemical structures of WFS and its structural analogues (B). 
Table 2 Determination of WFS in human serum using MIP/NPGL/GE and HPLC $(n=3)^{a_{1} c}$

\begin{tabular}{|c|c|c|c|c|c|}
\hline Method & Determined & Spiked $^{b}$ & Total found $^{b}$ & $\begin{array}{l}\text { Recovery } \\
(\%)\end{array}$ & RSD (\%) \\
\hline \multirow[t]{3}{*}{ HPLC } & - & 1.600 & $1.575 \pm 0.0262$ & 98.4 & 1.6 \\
\hline & - & 2.000 & $2.009 \pm 0.0066$ & 100.0 & 0.3 \\
\hline & - & 2.400 & $2.368 \pm 0.0216$ & 98.7 & 0.9 \\
\hline \multirow[t]{3}{*}{ WFS-MIP/NPGL/GE } & - & 1.600 & $1.659 \pm 0.0235$ & 103.7 & 1.4 \\
\hline & - & 2.000 & $2.099 \pm 0.0595$ & 104.9 & 2.8 \\
\hline & - & 2.400 & $2.471 \pm 0.1079$ & 102.9 & 4.3 \\
\hline
\end{tabular}

${ }^{a}$ Mean value \pm S.D. $^{b}$ The units of the value are $10^{-5} \mathrm{M}$ for HPLC and $10^{-9} \mathrm{M}$ for MIP/NPGL/GE. ${ }^{c}$ - not detected.

polymerization causes a thick MIP film on the electrode surface, and consequently many binding sites are buried in the polymeric matrix and become non-effective. As shown in Fig. 5B, the $\mathrm{MIP} / \mathrm{GE}$ with resorcinol as the monomer displayed the largest current response to WFS at the $\mathrm{pH}$ value of 7.5 and a $\mathrm{T}: \mathrm{M}$ of $1: 3$.

\subsection{Calibration curve and detection limit}

Under the optimized experimental conditions, the $\mathrm{CV}$ responses and calibration curve for detecting WFS using the proposed MIP/NPGL/GE sensor were investigated. Fig. 6 shows the correlation between the current shift $(\Delta I)$ and the logarithm of the concentration of WFS $(\ln C)$, and the corresponding linear regression equation is $\Delta I(\mu \mathrm{A})=5.2513 \ln C+129.6810$ $\left(R^{2}=0.9948\right)$ in the range of $1 \times 10^{-10}$ to $8 \times 10^{-8} \mathrm{M}$. The limit of detection (LOD) is down to $4.1 \times 10^{-11} \mathrm{M}(\mathrm{S} / \mathrm{N}=3)$, which is lower than the data from all of the reported WFS sensors we could find. This comparison is summarized in Table 1.

\subsection{Selectivity of the WFS-MIP/NPGL/GE}

In order to assess the specificity of the MIP-modified sensor, HCT, $V K_{4}$ and ASP, the structural analogues of WFS, were detected. As shown in Fig. 7, the MIP/NPGL/GE exhibited a much higher response to WFS than to the other analogues, whereas NIP/NPGL/GE shows no obvious difference in sensing all of the analytes. The $t$-test shown in Fig. 7A also proved the remarkable difference in the results of the sensor response of MIP/NPGL/GE towards the analogues and WFS with a significant level of 0.01 . The high specificity of MIP can be ascribed to the classic molecular imprinting effect. ${ }^{\mathbf{4 1 , 4 2}}$ In particular, during MIP preparation, WFS was incorporated into polymeric networks via several kinds of non-covalent interactions and the following removal of WFS left behind the imprinting cavities that are complementary to the template in size, shape and functionality. NIP, due to the lack of imprinting effect, can only yield non-specific binding toward different substances. The calibration curves of WFS and its structural analogues in the range of $1 \times 10^{-10}$ to $8 \times 10^{-8} \mathrm{M}$ are shown in Fig. S5, $\dagger$ in order to further investigate the sensitivity of MIP/NPGL/GE towards different compounds. It is obvious that MIP/NPGL/GE has a higher current shift for WFS than the analogues with the slopes of WFS (0.0531) being 3 times larger than that of HCT (0.0139), $\mathrm{VK}_{4}(0.0150)$ and ASP (0.0173). In addition, it is known that some co-existing ions may cause interference in WFS detection in real samples. Hence, a mixture of WFS and several ions $\left(\mathrm{NH}_{4}^{+}, \mathrm{Ca}^{2+}, \mathrm{Na}^{+}, \mathrm{K}^{+}, \mathrm{NO}_{3}{ }^{-}, \mathrm{SO}_{4}{ }^{2-}\right.$ and $\left.\mathrm{Cl}^{-}\right)$was assayed with the concentration of these ions being ten times higher than that of WFS. It was found that the sensing response from WFS alone is $97.8 \%$ of the response from the mixture, implying an excellent anti-interfering ability of MIP/NPGL/GE even in a complicated matrix where the amount of interferents is remarkably higher than the analyte.

\subsection{Repeatability and stability}

WFS at three different concentrations $\left(8 \times 10^{-8}, 8 \times 10^{-9}\right.$ and 8 $\times 10^{-10} \mathrm{M}$ ) was measured in triplicate using the same MIP/ NPGL/GE sensor. A relative standard deviation (RSD) of less than $2.5 \%$ was obtained, which reflects the good repeatability of the sensor. When not in use, all of the electrodes were stored in open air at room temperature. Compared with the initial electro-signal, the current response decreased by about 3\% over the first week, then little change was observed in a one-month period of time, exhibiting the decent stability of the hybrid electrode, which could be ascribed to the inherent stability of NPGL and MIP.

\subsection{Real sample analysis}

In order to evaluate the capability of the developed sensor in detecting real clinical samples, WFS in human blood was tested. The results shown in Table 2 exhibited satisfying accuracy with recoveries ranging from $102.9 \%$ to $104.9 \%$. In addition, HPLC was employed as the standard method to analyze the same blood samples and the results were in good accordance with those from our sensor, which proved the validity of the newly developed approach.

\section{Conclusions}

In this study, a novel hybrid sensor system was established using a two-step coating tactic of integrating NPGL modification and in situ MIP electro-synthesis for the specific detection of WFS. This is to our knowledge the first report on the combination of WFS-imprinted polymeric film with NPGL for establishing a hybrid membrane-modified electrochemical sensor. First of all, a 3D open and continuous nanoporous skeleton of NPGL helps to enhance electron transmission on one hand and 
allow for an enlarged platform for MIP loading on the other. Secondly, the MIP layer provides a number of imprinting cavities that match the template molecules based on geometrical size and the number and the steric arrangement of functional groups, guaranteeing specific recognition toward WFS. Application of the modified electrode in real sample analysis is proven to be more sensitive and convenient than the reference method, HPLC, with comparable accuracy and repeatability. Compared with the reported work of other systems, the sensor fabrication technique is facile and highly controllable, ensuring very good reproducibility and ease for developing admirable sensors in mass production. In summary, the sensor preparation procedure is simple and cost-effective, and the detection of WFS is far quicker than the commonly employed method, HPLC. Therefore, it can be expected that the developed sensor has great potential in therapeutic drug monitoring.

\section{Acknowledgements}

The project was financially supported by the National Natural Science Foundation of China $(81260487,81460543)$, and the Pairing Program of Shihezi University with Eminent Scholar in Elite University (SDJDZ201502). The authors would like to express their sincere appreciation to the Deanship of Scientific Research at King Saud University for its funding of this research through the Research Group Project No. Prolific Research Group No. 1436-011.

\section{Notes and references}

1 D. M. Ufer, Clin. Pharmacokinet., 2005, 44, 1227-1246.

2 R. Hull, G. Raskob, G. Pineo, D. Rosenbloom, W. Evans, T. Mallory, K. Anquist, F. Smith, G. Hughes and D. Green, N. Engl. J. Med., 1993, 329, 1370-1376.

3 R. Hull, T. Delmore, E. Genton, J. Hirsh, M. Gent, D. Sackett, D. Mcloughlin and P. Armstrong, N. Engl. J. Med., 1979, 301, 855-858.

4 L. Wallentin, S. Yusuf, M. D. Ezekowitz, M. Alings, M. Flather, M. G. Franzosi, P. Pais, A. Dans, J. Eikelboom and J. Oldgren, Lancet, 2010, 376, 975-983.

5 C. Banfield and M. Rowland, J. Pharm. Sci., 1984, 73, 13921396.

6 P. R. Ring and J. M. Bostick, J. Pharm. Biomed. Anal., 2000, 22, 573-581.

7 M. J. Kwon, H. J. Kim, J. W. Kim, K. H. Lee, K. H. Sohn, H. J. Cho, Y. K. On, J. S. Kim and S. Y. Lee, Korean J. Lab. Med., 2009, 29, 515-523.

8 W. P. Yau and E. Chan, J. Pharm. Biomed. Anal., 2002, 28, 107-123.

9 P. Y. Chen, P. C. Nien, C. W. Hu and K. C. Ho, Sens. Actuators, $B, 2010,146,466-471$.

10 M. A. Radwan, G. A. Bawazeer, N. M. Aloudah, B. T. AlQuadeib and H. Y. Aboul-Enein, Biomed. Chromatogr., 2012, 26, 6-11.

11 X. L. Chai, X. G. Zhou, A. W. Zhu, L. M. Zhang, Y. Qin, G. Y. Shi and Y. Tian, Angew. Chem., Int. Ed., 2013, 52, 8129-8133.
12 C. Zheng, X. L. Zhang, W. Liu, B. Liu, H. H. Yang, Z. A. Lin and G. N. Chen, Adv. Mater., 2013, 25, 5922-5927.

13 W. J. Lian, S. Liu, J. H. Yu, J. Li, M. Cui, W. Xu and J. D. Huang, Biosens. Bioelectron., 2013, 44, 70-76.

14 X. B. Zhang, Z. D. Wang, H. Xing, Y. Xiang and Y. Lu, Anal. Chem., 2010, 82, 5005-5011.

15 M. L. Yola, T. Eren and N. Atar, Sens. Actuators, B, 2014, 195, 28-32.

16 X. Yi, J. Huang-Xian and C. Hong-Yuan, Anal. Biochem., 2000, 278, 22-28.

17 M. L. Yola, T. Eren and N. Atar, Biosens. Bioelectron., 2014, 60, 277-285.

18 A. Salimi, M. Izadi, R. Hallaj and M. Rashidi, Electroanalysis, 2007, 19, 1668-1676.

19 M. L. Yola, L. Uzun, N. Özaltın and A. Denizli, Talanta, 2014, 120, 318-324.

20 V. C. Sanz, M. L. Mena, A. González-Cortés, P. Yanez-Sedeno and J. Pingarrón, Anal. Chim. Acta, 2005, 528, 1-8.

21 Y. Li, X. Fan, J. Qi, J. Ji, S. Wang, G. Zhang and F. Zhang, Mater. Res. Bull., 2010, 45, 1413-1418.

22 Y. Liu, J. Liu, H. Tang, J. Liu, B. Xu, F. Yu and Y. C. Li, Sens. Actuators, B, 2015, 206, 647-652.

23 Y. C. Li, Y. Liu, J. Liu, J. Liu, H. Tang, C. Cao, D. Zhao and Y. Ding, Sci. Rep., 2015, 5, 7699-7706.

24 Y. C. Li, H. Song, L. Zhang, P. Zuo, B. C. Ye, J. Yao and W. Chen, Biosens. Bioelectron., 2016, 78, 308-314.

25 C. Ho, D. W. M. Sin, K. M. Wong and H. P. O. Tang, Anal. Chim. Acta, 2005, 530, 23-31.

26 X. X. Dong, M. Y. Li, N. N. Feng, Y. M. Sun, C. Yang and Z. L. Xu, RSC Adv., 2015, 5, 86485-86489.

27 K. Zangeneh Kamali, A. Pandikumar, G. Sivaraman, H. N. Lim, S. P. Wren, T. Sun and N. M. Huang, RSC Adv., 2015, 5, 17809-17816.

28 M. L. Yola, N. Atar and T. Eren, Sens. Actuators, B, 2014, 198, 70-76.

29 B. C. Yin, S. Wu, J. L. Ma and B. C. Ye, Biosens. Bioelectron., 2015, 68, 365-370.

30 V. K. Gupta, M. L. Yola, N. Özaltın, N. Atar and Z. Üstündağ, Electrochim. Acta, 2013, 112, 37-43.

31 M. D. Scanlon, S. K. Urszula, B. Serguei, M. A. Domhnall, L. Dónal, D. Yi and M. Edmond, Langmuir, 2012, 28, 22512261.

32 Y. Ding, Y. J. Kim and J. Erlebacher, Adv. Mater., 2004, 16, 1897-1900.

33 W. Qin, Y. F. Zhao, C. X. Xu, W. Dan, Y. Y. Cai, H. Jing, L. He, B. Du and M. H. Yang, Biosens. Bioelectron., 2011, 26, 37143718.

34 Y. B. Mollamahale, M. Ghorbani, M. Ghalkhani, M. Vossoughi and A. Dolati, Electrochim. Acta, 2013, 106, 288-292.

35 W. W. Tu, X. L. Fang, J. Lou and Z. H. Dai, Analyst, 2015, 140, 2603-2607.

36 M. R. Mahmoudian, W. J. Basirun, P. M. Woi, M. Sookhakian, R. Yousefi, H. Ghadimi and Y. Alias, Mater. Sci. Eng., C, 2016, 59, 500-508.

37 B. Rezaei, O. Rahmanian and A. A. Ensafi, Sens. Actuators, B, 2014, 196, 539-545. 
38 M. B. Gholivand and L. Mohammadi-Behzad, Mater. Sci. Eng., C, 2015, 57, 77-87.

39 M. B. Gholivand and L. Mohammadi-Behzad, Mater. Sci. Eng., C, 2015, 48, 235-324.
40 M. M. Ghoneim and A. Tawfik, Anal. Chim. Acta, 2004, 511, 63-69.

41 L. Q. Lin, Y. C. Li, Q. Fu, L. C. He, J. Zhang and Q. Q. Zhang, Polymer, 2006, 47, 3792-3798.

42 Y. Ge and P. F. Anthony, Chem.-Eur. J., 2009, 15, 8100-8107. 